\title{
Classical topological order of the Rys F-model and its breakdown in realistic spin ice: Topological sectors of Faraday loops
}

\author{
Cristiano Nisoli \\ Theoretical Division, Los Alamos National Laboratory, Los Alamos, NM, 87545, USA \\ PACS 05.20.-y - Classical Statistical Mechanics \\ PACS 75.50.Ee - Antiferromagnetic Materials \\ PACS 75.30.Kz - Magnetic Phase Transitions
}

\begin{abstract}
Both the Rys F-model and antiferromagnetic square ice posses the same ordered, antiferromagnetic ground state, but the ordering transition is of second order in the latter, and of infinite order in the former. To tie this difference to topological properties and their breakdown, we introduce a Faraday line representation where loops carry the energy and magnetization of the system. Because of the absence of monopoles in the F-model, its loops have distinct topological properties, absent in square ice, and which allow for a natural partition of its phase space into topological sectors. Then the Néel temperature corresponds to a transition from trivial to nontrivial topological sectors. Moreover, its zero susceptibility below a critical field is explained by the homotopy invariance of its magnetization. In spin ices, instead, monopoles destroy the homotopy invariance of the magnetization, and thus erase this rich topological structure. Consequently, even trivial loops can be magnetized, and their susceptibility is never zero.
\end{abstract}

Introduction. - In 1967, Lieb solved 1] the Rys Fmodel 2] demonstrating an antiferromagnetic transition of rather unusual features: it is of infinite order and there is an order parameter, also infinitely smooth [3]; finally, a critical field is needed to elicit magnetization below $T_{c}$.

Lieb's work predated by five years the results of Kosterlitz and Thouless (KT) [4], which tied the infinitely continuous KT transition to topological properties. But regrettably, the importance of the infinite continuity in the F-model was apparently not recognized at first, nor was it associated to anything topological. Lieb had employed a line representation which, while immensely clever in allowing for an exact solution via transfer matrix, is not particularly conducive to physical intuition and does not make explicit the topological features of the system.

Our aim is not to provide new exact solutions of the F-model. It is the opposite. We seek to make explicit the topological nature of the system by mapping it to intuitive yet rigorous "Faraday lines", and then use it to deduce heuristically the transition and the properties of the model.

Our Faraday lines carry all the relevant observables: energy, magnetization, parity, and $\mathbb{Z}_{2}$ symmetry breaking. In the F-model they are always directed loops, thus making magnetization an homotopy invariant. This, we show, explains the critical field for susceptibility. We submit that ordering corresponds to a transition between topological sectors of trivial and non-trivial loops. While our deductions are based on heuristic arguments, our mapping to Faraday lines and the consequent partition of the phase space in topological sectors are exact.

Various reasons motivate our conceptualization. Firstly, we wish to elucidate how infinitely continuum transitions are related to topological sectors in a well known system. Secondly, vertex models enjoy wide applicability and are currently studied 5 -14. Thirdly, and generally, one wonders if the very features that make many topological models compelling also make them physically unrealistic.

The F-model approximates well the low-energy physics of nanomagnetic artificial square ice [15-17] as well as of monolayer spin ice [18, 19. And yet, these realizations possess none of its special properties 2024 . Their transitions are innocuously second order 25 27. (as recently explored experimentally [28]), and their susceptibility is never zero. Our framework explains those differences: in realistic spin ice, monopoles are sink and sources of the Faraday lines, thus destroying the topological structure.

Six-vertex models and the F-model. - A sixvertex model [5-14] is a set of binary spins placed on the 
edges of a square lattice (Fig. 1) such that only the six icerule obeying vertices (two spins pointing in, two pointing out [29]) are allowed, denoted t-I and t-II of energies $\epsilon_{\mathrm{I}}, \epsilon_{\mathrm{I}}$.

The Rys F-model is a particular six-vertex model whose energies are $0=\epsilon_{\mathrm{I}}<\epsilon_{\mathrm{II}}$. A spin configuration has energy

$$
\mathcal{H}=\epsilon_{\mathrm{II}} N_{\mathrm{II}}
$$

where $N_{\text {II }}$ is the number of t-II vertices.

The F-model is invariant under the $\mathbb{Z}_{2}$ time reversal symmetry, parity symmetry $A \leftrightarrow B$ (where $\mathrm{A}, \mathrm{B}$ are the the alternating $A, B$ sublattices), and discretized translations. Its two ordered ground states are antiferromagnetic tessellations of t-I, have opposite staggered [3] order parameter $\psi= \pm 1$, and thus break the $\mathbb{Z}_{2}$ symmetry. Hence, one expects a continuous transition. In fact we know from the exact solution that the transition infinitely continuous with algebraic correlations for $T>T_{c}=\epsilon_{\mathrm{II}} / \ln 2$.

In the two-dimensional ice model, instead, $\epsilon_{\mathrm{I}}=\epsilon_{\mathrm{II}}$, there is no energy scale and thus no transition. The system mimics the degeneracy of water ice in a two-dimensional system and was also solved by Lieb 30. It also describes the ground state of degenerate square ice 31 and the infinite $T$ limit of the F-model

Faraday loops. - The crux of our approach consists in choosing the proper description for the magnetic texture. In the antiferromagnetic ground state, the local magnetization is non-zero, but its coarse graining over a vertex is zero. Therefore, instead of considering the elementary spins $\vec{S}_{i}$, we describe magnetization by assigning it to to the vertices $v$, as $\vec{M}_{v}$, such that $\sum_{i} \vec{S}_{i}=\sum_{v} \vec{M}_{v}$.

The t-I vertices are demagnetized, while t-II ones carry magnetization. Because of the topological constraints, the magnetic moments of t-II vertices are always joined into Faraday lines. Thus, Faraday lines carry the magnetization and energy of the system. Moreover, on a torus, they are always directed loops and can be distinguished by topological triviality and parity. A combination of parity and topology determines their chirality. Finally, they separate antiferromagnetic domains of opposite staggered order parameter. All of this we show below.

Consider $L_{x} \times L_{y}$ vertices on a torus $\left(L_{x}, L_{y}\right.$ even). We proceed on a torus for clarity, and in the thermodynamic limit our considerations are transferrable to the standard $2 \mathrm{D}$ system. There, the topological group of the torus corresponds to Faraday lines extending from and to infinity.

A t-II vertex can be represented by an arrow connecting the centers of the plaquettes whose spins impinge antiferromagnetically in the vertex, thus assigning to it the magnetization $M_{x}= \pm 1, M_{y}= \pm 1$ (Fig. 1a).

The following propositions can be verified directly:

(i) Any spin configuration can be mapped into a set of non-intersecting, directed Faraday loops: indeed, a square plaquette can support 0,2 , or 4 t-IIs on its vertices. If 2 , they can always be connected unambiguously. If 4 , which we call a pinch, they can be joined in two directed lines in
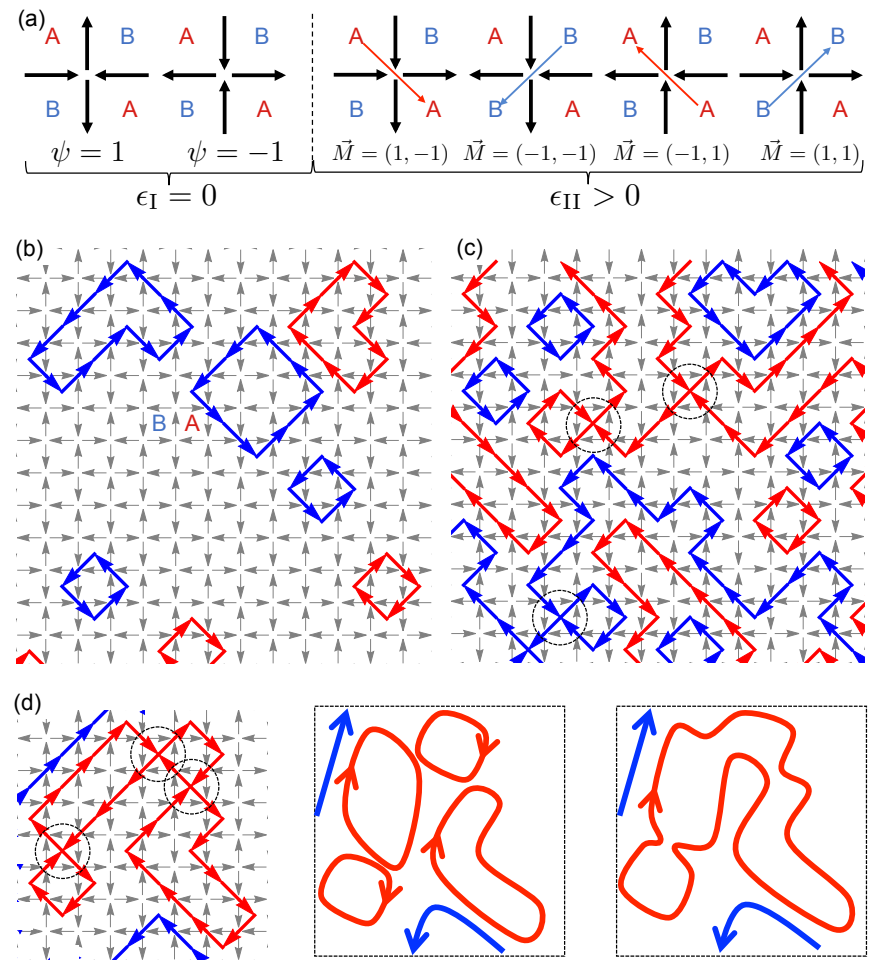

Fig. 1: (a) The six ice-rule obeying vertices. t-Is (left of the dotted line) possess a staggered antiferromagnetic order parameter $\psi= \pm 1$. t-IIs carry energy and magnetic moment. Connecting their net moments (red-blue diagonal arrows for A-B parity of the plaquettes they join), we obtain a directed loop-representation. (b-c) Loops in low (b) and high (c) energy configurations have defined parity and separate antiferromagnetic domains. (d) A spin configuration with 3 pinch points and 2 of its $2^{3}=8$ loop-representations.

two ways. For a configuration with $P$ pinches, there are $2^{P}$ loop-representations (Fig. 1b-d).

(ii) Loops have a defined parity: with the usual alternating $A / B$ assignment of plaquette parity, a loop will only cross either $A$ or $B$ plaquettes.

(iii) $A$ and $B$ loops (red and blue in Fig. 1) cannot cross.

(iv) The direction of loops is assigned thus: two nearby loops have the same (resp. opposite) direction if and only if they have same (resp. opposite) parity. If a loop is directly contained into another loop, the two have the same (resp. opposite) direction if and only if they have opposite (resp. same) parity.

Note (Fig. 1) that loops are also domain walls separating anti-ferrromagnetic domains with opposite sign of the staggered order parameter $\psi$. For completeness, we show in SI how the Faraday lines relate to the height formalism.

Modulo the pinch points, the spins-loops correspondence is bijective. Any set of directed loops drawn on the square lattice such that (i)-(iv) hold true corresponds to an acceptable spin configuration for the six-vertex model.

A trivial loop of the torus is one that can be contracted to zero. We say that a configuration is topologically nontrivial if at least one of his representation contains at least 
one non-trivial loop (and then their number must be even).

Faraday loops are the elementary excitations of the system, and the F-model is a loop gas. While only (and all) loops carry local magnetization, crucially, only topologically non-trivial loops carry net magnetization. Indeed, given a loop $\gamma$ made of vertices $v$, the total in-plane magnetization of the loop is

$$
\vec{M}_{\gamma}=\sum_{v \in \gamma} \vec{M}_{v}
$$

Then $\vec{M}_{\gamma}=0$ if and only if $\gamma$ is topologically trivial. If instead $\gamma$ wraps around the $x$ direction the net magnetization of the loops is $M_{y}=0, M_{x}= \pm L_{x} \hat{e}_{x}$ regardless of the length or shape of the loop.

We have reached a remarkable result: in the six-vertex model, magnetization is a homotopy invariant of the Faraday lines description. This implies that topologically trivial spin configurations have zero net magnetization, and do not couple with an external field. Therefore, the effect of a net Zeeman coupling can only be to induce topological transitions from trivial to non trivial configurations. This can be understood in terms of topological sectors.

Topological sectors. - We can now partition the phase space $\mathcal{P}$ (the set of all spin configurations) into topological sectors (subsets of defined topology).

Call $\mathcal{T}$ the sector of all topologically trivial configurations, and $\mathcal{W}$ its complementary. From Eq. (2), only configurations in $\mathcal{W}$ can have magnetization and we can further partition it accordingly.

We call a trivial (resp. non-trivial) elementary update of a spin configuration the flip of a trivial (resp. nontrivial) loop of spins that are all head to toe. Consider $n_{x}$ pairs of alternating $A$ and $B$ non-trivial loops in the direction $x$ (Fig. 2, second row, has $n=2$ ), with $0<$ $n_{x} \leq L_{y} / 2$. From (iv), their magnetization has the same direction. Call $\mathcal{M}_{ \pm n_{x} 0}$ the set of all topologically trivial updates of such configurations.

Because of homotopy invariance, trivial updates do not alter magnetization: from Eq. (2), each configuration in $\mathcal{M}_{n_{x} 0}$ carries magnetization $M_{y}=0, M_{x}=2 n_{x} L_{x}$ and magnetization density $m_{y}=0, m_{x}=2 n_{x} / L_{y}$. The same can be done to generate the sector $\mathcal{M}_{0 n_{y}}$ and for $\mathcal{M}_{n_{x} n_{y}}$, as the reader can verify via pairs of parallel helices.

Crucially, the union (which we call $\mathcal{M}$ ) of these magnetic sectors does not exhaust $\mathcal{W}$. Call $\mathcal{W}_{0}$ the set of nontrivial configurations that have zero net magnetization. We can partition $\mathcal{W}_{0}$ into: $\mathcal{W}_{A_{x} B_{x}}$ (resp. $\mathcal{W}_{A_{y} B_{y}}$ ), the sets of all configurations representable via non-trivial loops of type $A$ and $B$ in the $x$ (resp. $y$ ) direction; and $\mathcal{W}_{A_{x} A_{y}}$ (resp. $\mathcal{W}_{B_{x} B_{y}}$ ) the sets of all configurations representable via non-trivial loops of parity $A$ (resp. $B$ ) wrapping in both $x$ and $y$ directions (Fig. 2 bottom). Proposition (iii) forbids other sectors.

In summary, $\mathcal{P}$ is partitioned into $\mathcal{T}$ (trivial) and $\mathcal{W}$ (winding). $\mathcal{W}$ is partitioned into $\mathcal{M}$ (magnetic) and $\mathcal{W}_{0}$ (non magnetic). $\mathcal{W}_{0}$ is partitioned into $\mathcal{W}_{A_{x} A_{y}}$ (A loop
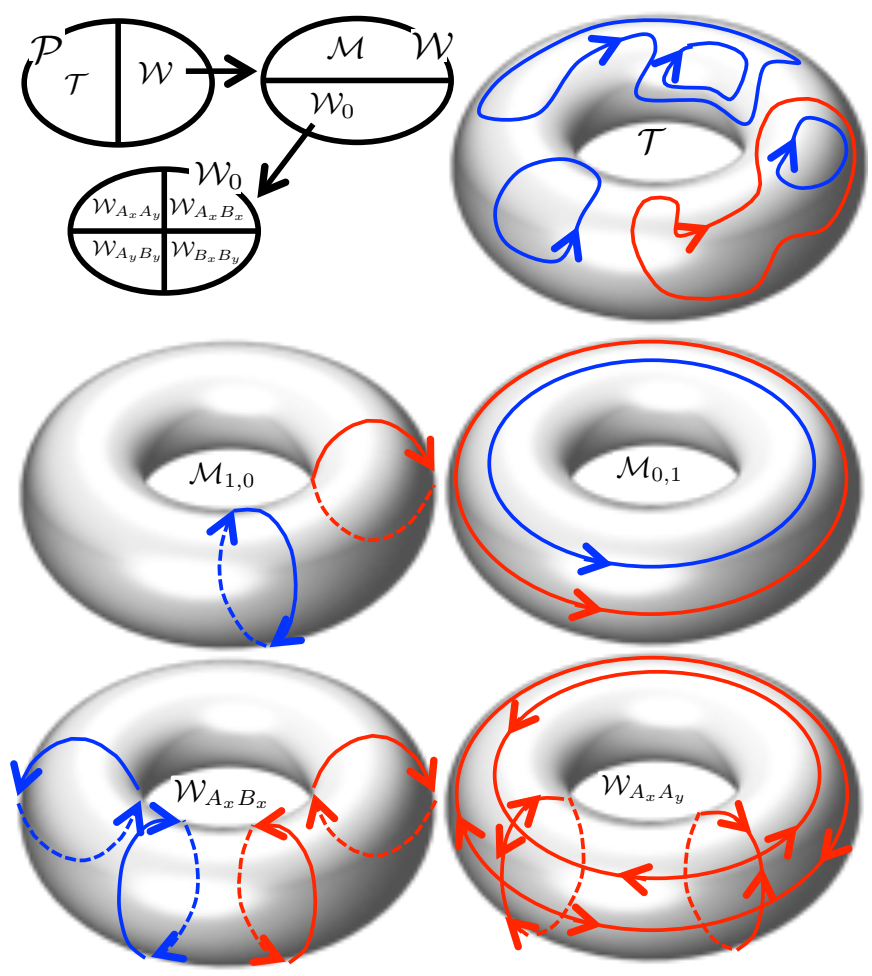

Fig. 2: Venn diagrams of the partition of the phase space into topological sectors and schematics for elements of $\mathcal{T}$ (top), $\mathcal{M}$ (middle), and $\mathcal{W}_{0}$ (bottom) represented on the torus. $\mathcal{P}$ is partitioned into $\mathcal{T}$ and $\mathcal{W}$, corresponding to trivial and non-trivial loops. $\mathcal{W}$ is partitioned into $\mathcal{M}$ (corresponding to magnetized configuration) and $\mathcal{W}_{0} . \mathcal{W}_{0}$ is partitioned into sectors corresponding to loops of different parity.

winding in both directions, and same for B) $\mathcal{W}_{A_{x} B_{x}}$ (A and $\mathrm{B}$ loops winding in the $\mathrm{x}$ direction, but alternating as to give zero magnetization, and same for $y$ ).

We can introduce winding topological order parameters, $w_{A}$ and $w_{B}$, for each parity. For a configuration $C$ and its (possibly multiple) loop-representation(s) $R$ made of loops $\gamma$ we define

$$
w_{A}(C)=\sup _{R \in C} \sum_{\gamma_{A} \in R}\left|\frac{1}{L_{x} L_{y}} \sum_{v \in \gamma_{A}} \vec{M}_{v}\right|
$$

the density of winding number of $A$ loops of the configuration $C$, and $w^{+}=w_{A}+w_{B}, w^{-}=w_{A}-w_{B}$.

Temperature transition. - When the phase space is partitioned into sectors $\mathcal{D} \subset \mathcal{P}$, we can call

$$
Z_{\mathcal{D}}(T)=\sum_{\mathcal{C} \in \mathcal{D}} \exp [-\mathcal{H}(\mathcal{C}) / T]
$$

whose sum is restricted to configurations in $\mathcal{D}$, the partition function of $\mathcal{D} . P_{\mathcal{D}}=Z_{\mathcal{D}} / Z$ is then the probability of finding the system in a configuration of the sector $\mathcal{D}$. Any observable is said to be limited to the sector $\mathcal{D}$ if obtained from $F_{\mathcal{D}}=-T \ln Z_{\mathcal{D}}$. The total partition function is the sum of the partition functions of the sectors. 
If $P_{\mathcal{D}} \rightarrow 1^{-}$in the thermodynamic limit (and thus $F \rightarrow$ $F_{\mathcal{D}}$ ), we say that the system is asymptotically confined to sector $\mathcal{D}$ and that the partition function projects to that sector. In this language, a phase transition corresponds to the system "switching" between different sectors of the phase space, to which it is confined in the thermodynamic limit. When those sectors are topologically distinct, we say that the transition is topological.

First we show that in absence of a field, the system is asymptotically confined to $\mathcal{T} \cup \mathcal{W}_{0}$, that is the complementary of $\mathcal{M}$. Indeed, consider $f(m, T)$, the density of free energy limited to the sector $\mathcal{M}_{m L_{y} / 2,0}$. Then $H_{x}=\partial_{m} f(m, T)$ is the magnetic field. $f(m)$ must be concave and even in $m$, thus has minimum at $m=0$.

Note that the thermal average of $\psi$ in $\mathcal{W}$ is zero. To prove it, consider e.g. $\mathcal{M}_{1,0}$. Its lowest energy state is degenerate, corresponding to one $A$ and one $B$ non-trivial loops each of length $L_{x}$, variously assigned, subdividing the torus in two domains of opposite $\psi$. Averaging $\psi$ over all those configurations then returns zero. The same argument can be replicated for any sector in $\mathcal{W}$.

That only $\mathcal{T}$ can exhibit long range order and thus $\psi \neq 0$ only in $\mathcal{T}$ is intuitive. Indeed, the symmetry breaking that leads to $\psi \neq 0$ is driven by the contraction of the domain walls (Faraday loops) as temperature is lowered because of their tensile strength. But outside of $\mathcal{T}$, by definition, there are always some non-contractible loops.

Thus, antiferromagnetic ordering in the absence of a field must correspond to a transition between the topological sectors $\mathcal{T}$ and $\mathcal{W}_{0}$. Consider a non-trivial loop of lowest energy winding around the $x$ axis. It has length $L_{x}$ and degeneracy $\left(\begin{array}{c}L_{x} \\ L_{x} / 2\end{array}\right) \sim 2^{L_{x}}$, for large $L_{x}$. Its free energy is then

$$
\Delta F=L_{x}\left(\epsilon_{\mathrm{II}}-T \ln 2\right),
$$

and goes to $-\infty(+\infty)$ in the thermodynamic limit for $T>T_{c}\left(T<T_{c}\right)$ with $T_{c}=\epsilon_{\mathrm{II}} / \ln 2$.

As in the heuristic argument of Kosterlitz and Thouless, Eq. (5) implies that above $T_{c}$ the system is asymptotically confined to the topologically non-trivial sector $\mathcal{W}_{0}$, where $\psi=0$, and below $T_{c}$ to the trivial $\mathcal{T}$ where $\psi \neq 0$. Therefore, $T_{c}=\epsilon_{\mathrm{II}} / \ln 2$ is the Néel temperature. Crucially, it corresponds to the Néel temperature in Lieb's solution.

Low $T$ configurations correspond to an antiferromagnetic background decorated by Faraday loops (domain walls, Fig. 1b). As $T$ increases, loops grow and coalesce forming at $T_{c}$ a topologically non-trivial network (Fig. 1c), in a classical analogue to a string-net condensation 32].

There is more. We have seen that $\mathcal{T}$ hosts a $\mathbb{Z}_{2}$ symmetry breaking in the sign of $\psi$. But a topological symmetry breaking also exists in $\mathcal{W}$, between the topological sectors $\mathcal{W}_{A_{x} A_{y}}$ and $\mathcal{W}_{B_{x} B_{y}}$ as they have the same free energy but different parity. At $T>T_{c}$ the systems must choose whether the network of winding loops has parity $A$ or $B$, because loops of different parity cannot cross. This leads to a breaking of the $A \leftrightarrow B$ parity symmetry of the topologically non-trivial loops and thus in the sign of $w^{-}$.
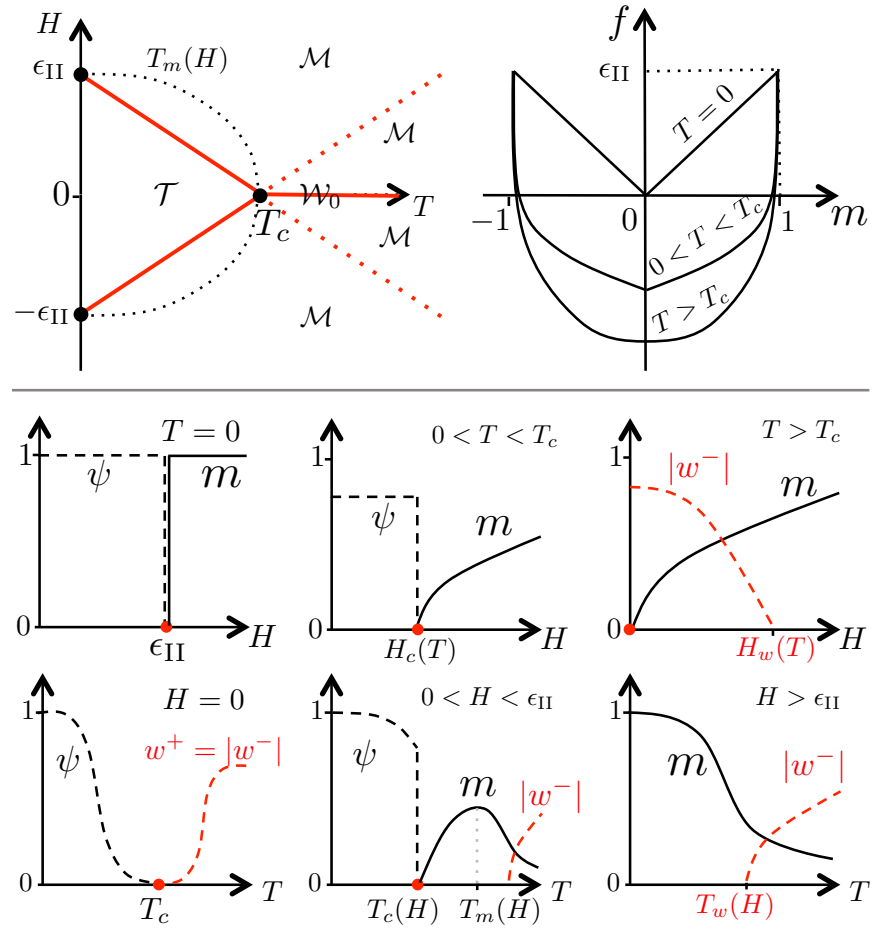

Fig. 3: First Panel: schematics of the phase diagram for an horizontal field $H$. The red line marks the distinction between topological sectors and is critical. On the line $H=0, T>T_{c}$ the system is confined to $\mathcal{W}_{0}$, with $m=0, \psi=0$, and it is critical. We sketch a possible $T_{m}(H)$ (black dotted) and the conjectured $T_{w}(H)$ (red dotted). Second panel: sketches of $f(m, T)$. The singularity in $m=0$ at $0 \leq T<T_{c}$ disappears at $T \geq T_{c}$. Other panels: sketches of $\psi, m, w^{-}$vs. $T$ and $H$ as deduced from the top two panels and considerations in text.

Thus, at $T<T_{c}$ we have $w_{A}=w_{B}=0, \psi>0$. At $T>T_{c}$ we have $\psi=0, w^{+}>0$ and $w^{-}= \pm w^{+}$. We suspect that $w^{+}, w^{-}$reach zero infinitely continuously as $T \rightarrow T_{c}^{+}$just like $\psi\left[3\right.$ does for $T \rightarrow T_{c}^{-}$, though we are incapable of predicting it within our framework.

Field induced transitions. - We also have transitions under field between demagnetized $\mathcal{T}$ and magnetized $\mathcal{M}$, and there $\psi$ is discontinuous except at $T=T_{c}$, as we show below.

Consider an horizontal field and let us study the shape of $f(m)$. At $T=0$, the free energy is trivially $f(m)=\epsilon_{\mathrm{II}} m$, and the curve of magnetization is a step function $(m=0$ for $H<\epsilon_{\mathrm{II}}$ and $m=1$ for $H>\epsilon_{\mathrm{II}}$ ).

The sector of saturated magnetization $\mathcal{W}_{L_{y} / 2,0}(m=1)$ contains configurations all of the same energy, where all the horizontal spins are pointing to the right, whereas half of the vertical rows point up and half down. Its entropy is subextensive [its degeneracy being $\left(\begin{array}{c}L_{y} \\ L_{y} / 2\end{array}\right)$ ], and therefore $f( \pm 1, T)=\epsilon_{\mathrm{II}}$ at any temperature.

For $m \simeq 0$, at $0<T<T_{c}$ entropy favors configurations in which $m L_{y} / 2$ horizontal, non-trivial loops of alternating parity and of magnetization aligned to the left are 
maximally spaced at a distance $1 /|m|$, arbitrarily large. The free energy can thus be approximated by a trivial term from the bulk plus a non-trivial contribution from the loops, or from Eq. (5)

$$
f(m) \simeq f_{\mathcal{T}}+|m|\left(\epsilon_{\mathrm{II}}-T \ln 2\right)+o(m) \text { for } m \rightarrow 0 .
$$

The weak singularity in $m=0$ implies a critical field

$$
\left|H_{c}(T)\right|=\epsilon_{\mathrm{II}}-T \ln 2
$$

below which the system is asymptotically confined to $\mathcal{T}$ and there is no magnetization.

Instead, no critical field exist when $T>T_{c}$. In such case, there are topologically non-trivial loops even at $m=$ 0 and thus no singularity in $f(m)$. Intuitively, the noncontractible loops present above criticality can be biased by weak fields to be of the proper alternation of parity.

Fig. 3 summarizes our results. The top panel, left, shows the phase diagram expressed in terms of topological sectors. Top panel, right sketches $f(m)$ at different temperatures, from which curves for $m$ and $\psi$ can be obtained qualitatively (bottom panels).

When $0<H<H_{c}(T), 0 \leq T<T_{c}$ the system is asymptotically confined to $\mathcal{T}$, and the magnetic field has no effect on the free energy. Thus, $\psi$ drops discontinuously to zero across the critical line (red) as the system switches to the sectors in $\mathcal{M}$ and magnetization develops. The entire line $H=0, T \geq T_{c}$ corresponds to the system being confined to $\mathcal{W}_{0}$ and is critical (with algebraic correlations 5 ).

When $0<H<\epsilon_{\mathrm{II}}$, the magnetic moment is nonmonotonic in temperature (Fig. 3) because as $T$ increases and crosses the critical line, magnetization ensues, yet for large $T$ it must tend to zero. We call $T_{m}=T_{m}(H)$ the temperature at which the maximum of $m$ is achieved. We show in SI that $T_{m} \rightarrow 0$ when $H \rightarrow \epsilon_{\mathrm{II}}^{-}$, while $T_{m} \rightarrow T_{c}^{-}$ when $H \rightarrow 0$. A plausible sketch of $T_{m}(H)$ is shown in Fig. 3.

Configurations in $\mathcal{T}_{0}$ always have $w^{+}=w^{-}=0$. Configurations in $\mathcal{W}_{0}$ always have $w^{+}>0$, while $w^{-}$can be zero, e.g. in $\mathcal{W}_{A_{x} B_{x}}$. It is always $\left|w^{-}\right|=w^{+}\left(\operatorname{resp} w^{-}=-w^{+}\right)$ in $\mathcal{W}_{A_{x} A_{y}}$ (resp. $\mathcal{W}_{B_{x} B_{y}}$ ). For a configuration with density of magnetization $m$, we have $w^{+} \geq m$. Instead, $w^{-}$ can be zero, and is indeed zero at minimal energy where non-trivial loops perfectly alternate parity. Configurations of higher energy can have $w^{-}>0$ when loops point in the direction opposite to the magnetization, breaking the parity alternation (and thus $w^{+}>m$ ). We conjecture that such loops are possible only when their free energy (inclusive of a Zeeman term) is negative, or $\epsilon_{\mathrm{II}}+|H|-T \ln 2<0$, leading to a new line $T_{w}(H)=\left(\epsilon_{\mathrm{II}}+|H|\right) / \ln 2$, for the appearance of the winding order parameter $w^{-}$. In Fig. 3 we have sketched possible curves for $w^{-}$(red dashed line).

Monopoles and Faraday lines in spin ice. - Any kinetics of the F-model must involve topologically-trivial updates only, at least in the thermodynamic limit. But
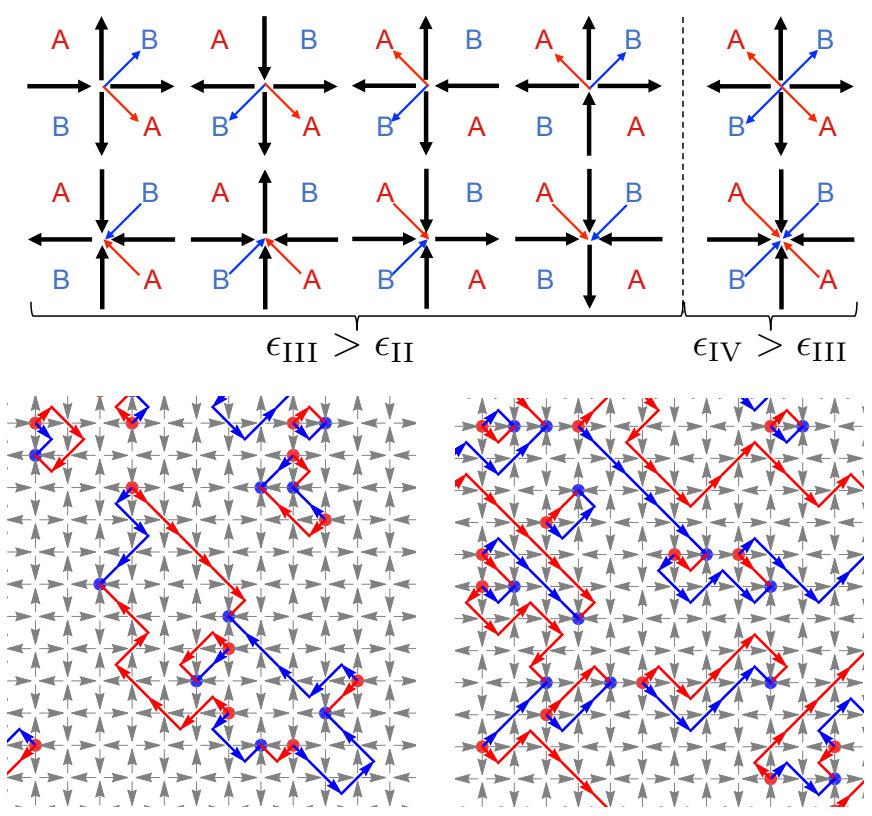

Fig. 4: Top: The ten extra vertices (monopoles) included in the sixteen-vertex model can also be represented by arrows separating frustrated spins and whose sum represents the total magnetization. The t-III (left of dotted line) have topological charges \pm 2 , magnetizations $\vec{M}=( \pm 1,0),(0, \pm 1)$, and energy $\epsilon_{\mathrm{III}}>\epsilon_{\mathrm{II}}$. t-IV vertices have topological charges \pm 4 , zero magnetization, and energy $\epsilon_{\mathrm{IV}}>\epsilon_{\mathrm{III}}$. For all, $\psi=0$. In the resulting loops representation (bottom left), domain wall-loops mix parity and invert magnetization at the monopoles, (red and blue dots for negative and positive respectively). Hence, topologically trivial loops can carry net magnetization (Bottom right shows horizontal magnetization).

sectors in $\mathcal{M}$ differ by non-trivial updates. This assures ergodicity breaking [33]. We find therefore the unphysical conclusion that the system will persist in a magnetized, high energy state forever after the field is removed. Also, its magnetization will be independent on changes of temperature (though its free energy would change).

This indeed does not happen in real systems such as square spin ices $15,16,34$, which instead evolve via individual spin flips, which are forbidden in the F-model as they lead to ice-rule violations, or monopoles.

At nearest neighbors, square spin ice is described by the sixteen-vertex model 25], which contains all the possible vertices. Figure 4 shows the ten extra vertices, as t-III and t-IV ice rule violating vertices of energies $\epsilon_{\mathrm{II}}<\epsilon_{\mathrm{III}}<\epsilon_{\mathrm{IV}}$, endowed with a topological charge $( \pm 2, \pm 4)$ defined as the difference of spins pointing in and out. This energy hierarchy describes the most common magnetic realizations $15,20,21,26,28,35,36$ and also particle-based ices $22,24,37$ via a proper mapping at equilibrium [38, 39], though even different hierarchies can be obtained through various clever methods $31,40,42$.

Figure 4 shows that monopoles too can be incorporated into a Faraday picture, but modify it essentially. Now 
monopoles allow for the mixing of $A$ and $B$ lines, and thus there is no longer parity distinction for the loops. Also, we see that Faraday lines can now be just lines and not necessarily loops, and monopoles are their sinks and sources. This is the a geometric version of the Gauss' law.

Faraday lines still compose into domain wall loops, These can contain an even number of \pm 2 monopoles, whose charge alternates in sign along the loop. Therefore, magnetization is no longer an homotopy invariant of non-contractible loops. Topologically trivial loops can carry net magnetization if they contain monopoles.

Therefore, the previous partition of the phase space in topological sectors breaks down. And indeed, the antiferromagnetic transition in square ice is known to be of second order, as the system can be mapped into a frustrated $J_{1}-J_{2}$ Ising model 25$]$. Also, by allowing for more entropy to destroy the ordered state, monopoles lower the critical temperature, or $T_{c}<\epsilon_{\mathrm{II}} / \ln 2$.

We can also understand in this picture why there is not any critical field for magnetization. The domain wall themselves, if they contain monopoles, are magnetizable. Because the magnetization flips verse at the monopole, a trivial loop can carry net magnetization. An external field can align the magnetization of a trivial loop by reallocating its monopoles along the domain wall, and thus the system is magnetically susceptible at any field even in the antiferromagnetic phase. There will still be a critical field $H_{c}(T)$ for the disappearance of $\psi$ [and clearly $H_{c}(0)=\epsilon_{\mathrm{II}}, H_{c}\left(T_{c}\right)=0$ ] but the system can be magnetized for $H<H_{c}(T)$ via the paramagnetism of the domain wall loops (Fig. 5), proportional to the density of t-II. Thus magnetization should scale as $m \sim H \exp \left(-\epsilon_{\mathrm{II}} / T\right) / T$.

Finally, the kinetics of real spin ice loses the topological ergodicity breaking of the Rys F-model precisely because any kinetics is monopole kinetics: a single spin flip corresponds to either creation-annihilation of a monopole pair or to its motion. In turn, this implies to the nucleation of loops and also their growth, contraction, or fluctuation, as it will be shown in a future work.

Faraday Lines and Dirac strings. - This representation is useful also when the square ice is degenerate $31,40,42]$, or $\epsilon_{I}=\epsilon_{\mathrm{II}}<\epsilon_{\mathrm{III}}<\epsilon_{\mathrm{IV}}$. There, the ground state is described by the two-dimensional ice model explained above: monopoles are absent, the topological structure described above is valid, and the system is asymptottically confined to the sector $\mathcal{W}_{0}$ at zero applied field and to $\mathcal{M}$ at any non zero field. It has therefore non-zero susceptibility at any field.

However, at any non-zero temperature monopole forms as sources and sinks of the Faraday lines. Therefore the $T=0$ case cannot be considered a $T \downarrow 0$ limit for degenerate square spin ice, as it is essentially topologically different. In degenerate square spin ice, $T=0$ is therefore the essentially non-perturbative critical point for a topological phase, thus explaining why the correlation length $\xi$ tends to infinity faster than algebraically [43,44. More-
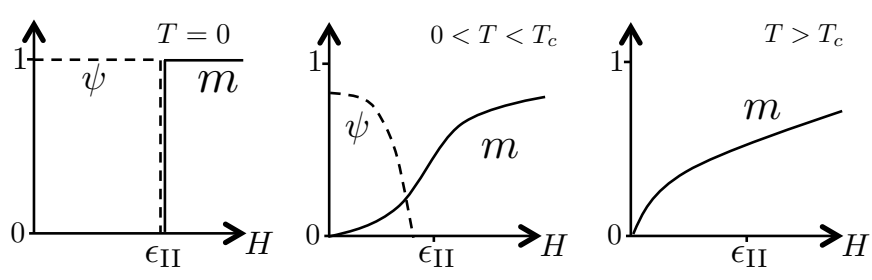

Fig. 5: Sketches of $m$ and $\psi$ in a square ice. At $T=0$ we have the usual step function as a pointwise limit. The case below $T_{c}$ differs considerably from the F-model. There is small but nonzero susceptibility at low field, and the maximum susceptibility corresponds to a left neighborhood of $\epsilon_{\mathrm{II}}$ where $\psi=0$.

over, in physical realizations the entire model generally breaks down at low temperature in real systems.

In future work, we will treat more in depth the the relationship between Faraday lines, the so-called Dirac strings, the gauge freedom associated to the height function representation, and magnetic fragmentation [45 47].

Here we note that for an antiferromagnetic square ice the language of Dirac strings is often unsuitable. It is often said that monopoles in square ice are "linearly confined" by Dirac strings. That is in general not true.

Looking at Fig 4 the reader can verify that two monopoles connected by Faraday lines can feel no force bringing them together - or indeed no force at all: it is generally not true that a spin always exists, impinging on a given monopole, whose flipping reduces the energy by moving the monopole. Certainly, Faraday lines have tensile strength, but that is true generally in the F-model where monopoles are absent.

We can talk about Dirac strings of tensile strength merely in the case in which a monopole pair can be annihilated on a t-I antiferromagnetic tessellation by flipping a single directed line of spins. While interesting, that does not describe, however, all the possible situations. Furthermore, such a case is still better described by two Faraday lines running parallel to the claimed Dirac string, both starting from the negative monopoles and ending in the positive one (many examples are visible in Fig. 4), as Faraday lines unequivocally carry energy and magnetization.

Conclusion. - We have introduced a Faraday Line picture for square ice in general, and the six-vertex model in particular. It allows for a partition of the phase space into topological sections, shedding light on the topological nature of the transitions of the Rys F-model that. In spin ice materials this representation survives, but its topological features break down because of monopoles and the Gauss' law. Within this picture, it would be interesting to investigate scaling limits in which the transition in spin ice tends to the topological. Furthermore, our picture of topological non-trivial loops can be employed in finitesize realizations of artificial spin ice on a cylinder, which are now possible [48, or in attacking problems of the sixvertex model with fixed boundary conditions $[5,14]$. 


$$
* * *
$$

We thank C. Castelnovo (Cambridge) and C. Batista (Tennessee) for useful feedback. This work was carried out under the auspices of the U.S. DoE through the Los Alamos National Laboratory, operated by Triad National Security, LLC (Contract No. 892333218NCA000001).

\section{REFERENCES}

[1] Lieb E. H., Physical Review Letters, 18 (1967) 1046.

[2] Rys F., Helvetica Physica Acta, 36 (1963) 537.

[3] Baxter R. J., Journal of Statistical Physics, 9 (1973) 145.

[4] Kosterlitz J. M. and Thouless D. J., Journal of Physics C: Solid State Physics, 6 (1973) 1181.

[5] BAXTER R., Exactly solved models in statistical mechanics (Academic, New York) 1982.

[6] Bogoliubov N., Pronko A. and Zvonarev M., Journal of Physics A: Mathematical and General, 35 (2002) 5525.

[7] Pannevis N. B., Critical behaviour of the six vertex $f$ model Master's thesis (2012).

[8] Zinn-Justin P., Physical Review E, 62 (2000) 3411.

[9] Zinn-Justin P., arXiv preprint arXiv:0901.0665, (2009)

[10] Barkema G. and Newman M., Physical Review E, 57 (1998) 1155.

[11] Weigel M. and Janke W., Journal of Physics A: Mathematical and General, 38 (2005) 7067.

[12] Keesman R. and Lamers J., Physical Review E, 95 (2017) 052117.

[13] EloRAnta K., Journal of statistical physics, 96 (1999) 1091.

[14] van Beijeren H., Physical Review Letters, 38 (1977) 993.

[15] Wang R. F., Nisoli C., Freitas R. S., Li J., MCConville W., Cooley B. J., Lund M. S., Samarth N., Leighton C., Crespi V. H. and Schiffer P., $\mathrm{Na}$ ture, 439 (2006) 303.

[16] Nisoli C., Moessner R. and Schiffer P., Reviews of Modern Physics, 85 (2013) 1473.

[17] Gilbert I., Nisoli C. and Schiffer P., Physics Today, 69 (2016) 54.

[18] Bovo L., Rouleau C., Prabhakaran D. and Bramwell S., Nature communications, 10 (2019) 1219.

[19] Jaubert L., Lin T., Opel T., Holdsworth P. and Gingras M., Physical review letters, 118 (2017) 207206.

[20] Porro J., Bedoya-Pinto A., Berger A. and VavasSORI P., New Journal of Physics, 15 (2013) 055012.

[21] Zhang S., Gilbert I., Nisoli C., Chern G.-W., ErICKSON M. J., OB́rien L., Leighton C., LAMMERT P. E., Crespi V. H. and Schiffer P., Nature, 500 (2013) 553.

[22] Libál A., Reichhardt C. and Olson Reichhardt C. J., Phys. Rev. Lett., 97 (2006) 228302.

[23] Ortiz-Ambriz A. and Tierno P., Nature communications, 7 (2016) .

[24] Libál A., Nisoli C., Reichhardt C. J. O. and ReichHARDt C., Phys. Rev. Lett., 120 (2018) 027204.

[25] Wu F. Y., Phys. Rev. Lett., 22 (1969) 1174.
[26] Levis D., Cugliandolo L. F., Foini L. and Tarzia M., Physical review letters, 110 (2013) 207206.

[27] Cugliandolo L. F., Journal of Statistical Physics, (2017) 1.

[28] Sendetskyi O., Scagnoli V., Leo N., Anghinolfi L., Alberca A., Lüning J., Staub U., Derlet P. M. and HeYdERMAN L. J., arXiv preprint arXiv:1905.07246, (2019) .

[29] Bernal J. and Fowler R., The Journal of Chemical Physics, 1 (1933) 515.

[30] Lieb E. H., Physical Review, 162 (1967) 162.

[31] Perrin Y., Canals B. and Rougemaille N., Nature, 540 (2016) 410.

[32] Levin M. A. and Wen X.-G., Physical Review B, 71 (2005) 045110.

[33] Palmer R., Advances in Physics, 31 (1982) 669.

[34] Ramirez A. P., Hayashi A., Cava R. J., Siddharthan R. and Shastry B. S., Nature, 399 (1999) 333.

[35] Morgan J. P., Stein A., Langridge S. and Marrows C. H., Nat. Phys., 7 (2010) 75.

[36] Nisoli C., Li J., Ke X., Garand D., Schiffer P. and Crespi V. H., Phys. Rev. Lett., 105 (2010) 047205.

[37] Libál A., Lee D. Y., Ortiz-Ambriz A., Reichhardt C., Reichhardt C. J., Tierno P. and Nisoli C., Nature communications, 9 (2018) 4146.

[38] Nisoli C., New Journal of Physics, 16 (2014) 113049.

[39] Nisoli C., Physical Review Letters, 120 (2018) 167205.

[40] Möller G. and Moessner R., Phys. Rev. B, 80 (2009) 140409.

[41] Farhan A., Saccone M., Petersen C. F., Dhuey S., Chopdekar R. V., Huang Y.-L., Kent N., Chen Z., Alava M. J., Lippert T. et al., Science advances, $\mathbf{5}$ (2019) eaav6380.

[42] Östman E., Stopfel H., Chiohr I.-A., Arnalds U. B., Stein A., Kapaklis V. and Huörvarsson B., Nature Physics, 14 (2018) 375.

[43] Nisoli C., arXiv preprint arXiv:2004.03735, (2020).

[44] Fennell T., Deen P., Wildes A., Schmalzl K., Prabhakaran D., Boothroyd A., Aldus R., MCMorrow D. and Bramwell S., Science, 326 (2009) 415.

[45] Brooks-Bartlett M., Banks S. T., Jaubert L. D., Harman-Clarke A. and Holdsworth P. C., Physical Review X, 4 (2014) 011007.

[46] Canals B., Chioar I.-A., Nguyen V.-D., Hehn M., Lacour D., Montaigne F., Locatelli A., Mentes T. O., Burgos B. S. and Rougemaille N., Nature communications, 7 (2016) .

[47] Petit S., Lhotel E., Canals B., Hatnean M. C., OlLivier J., Mutka H., Ressouche E., Wildes A., Lees M. and Balakrishnan G., Nature Physics, 12 (2016) 746.

[48] Gliga S., Seniutinas G., Weber A. and David C., Materials Today, 26 (2019) 100. 